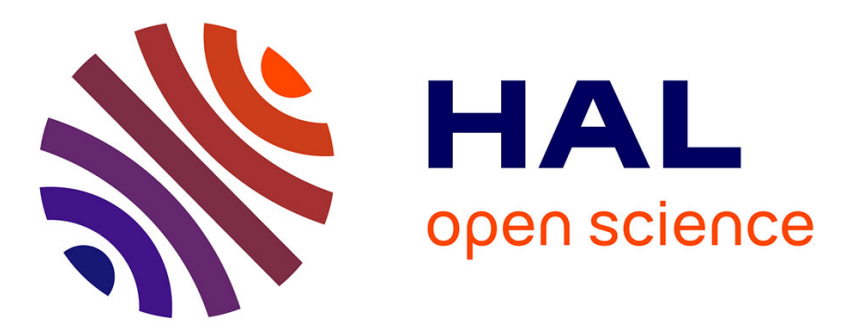

\title{
A Minimum-Fuel Fixed-Time Low-Thrust Rendezvous Solved with the Switching Systems Theory
}

Clément Gazzino, Denis Arzelier, Luca Cerri, Damiana Losa, Christophe Louembet, Christelle Pittet

\section{- To cite this version:}

Clément Gazzino, Denis Arzelier, Luca Cerri, Damiana Losa, Christophe Louembet, et al.. A Minimum-Fuel Fixed-Time Low-Thrust Rendezvous Solved with the Switching Systems Theory. Transactions of the Japan Society for Aeronautical and Space Sciences, 2018, ISTS Special Issue, 16 (7), pp.628-634. 10.2322/tastj.16.628 . hal-01609252

\section{HAL Id: hal-01609252 \\ https://hal.laas.fr/hal-01609252}

Submitted on 16 Oct 2017

HAL is a multi-disciplinary open access archive for the deposit and dissemination of scientific research documents, whether they are published or not. The documents may come from teaching and research institutions in France or abroad, or from public or private research centers.
L'archive ouverte pluridisciplinaire HAL, est destinée au dépôt et à la diffusion de documents scientifiques de niveau recherche, publiés ou non, émanant des établissements d'enseignement et de recherche français ou étrangers, des laboratoires publics ou privés. 


\title{
A Minimum-Fuel Fixed-Time Low-Thrust Rendezvous Solved with the Switching Systems Theory
}

\author{
By Clément Gazzino, ${ }^{1)}$ Denis Arzelier,${ }^{1)}$ Luca Cerri,,${ }^{2)}$ Damiana Losa,${ }^{3)}$ Christophe Louembet, ${ }^{1)}$ and Christelle PitTet ${ }^{2)}$ \\ 1) LAAS-CNRS, Université de Toulouse, CNRS, Toulouse, France \\ ${ }^{2)}$ CNES, Centre Spatial de Toulouse, Toulouse, France \\ ${ }^{3)}$ Thales Alenia Space, Cannes la Bocca, France
}

\begin{abstract}
In this paper, a fuel optimal rendezvous problem is tackled in the Hill-Clohessy-Wiltshire framework with several operational constraints as bounds on the thrust, non linear non convex and disjunctive operational constraints (on-off profile of the thrusters, minimum elapsed time between two consecutive firings...). An indirect method and a decomposition technique have already been combined in order to solve this kind of optimal control problem with such constraints. Due to a great number of parameters to tune, satisfactory results are hard to obtain and are sensitive to the initial condition. Assuming that no singular arc exists, it can be shown that the optimal control exhibits a bang-bang structure whose optimal switching times are to be found. Noticing that a system with a bang-bang control profile can be considered as two subsystems switching from one with control on to with control off, and vice-versa, a technique coming from the switching systems theory is used in order to optimise the switching times.
\end{abstract}

Key Words: Satellite Rendezvous, Low Thrust, Optimal Control, Switching Systems

$\begin{array}{ccll}\text { Nomenclature } & \\ F_{\text {max }} & : & \text { maximum level of thrust } \\ n & : & \text { mean motion of the reference orbit } \\ \text { OCP } & : & \text { Optimal Control Problem } \\ P & : & \text { total number of thrusts } \\ P_{i} & : & \text { number of thrusts for thruster } i \\ t_{i, j} & : & \text { middle time of the } j^{\text {th }} \text { thrust of thruster } i \\ T_{d} & : & \text { minimum duration between two thrusts of } \\ & & \text { two different thrusters } \\ T_{k} & : & \text { coast or thrust interval } \\ T_{l} & : & \text { minimum duration of a thrust } \\ \text { TPBVP } & : & \text { Two-Point Boundary Value Problem } \\ T_{s} & : & \text { minimum duration between two thrusts of } \\ & & \text { the same thruster } \\ u & : & \text { control vector } \\ U_{k} & : & \text { admissible control on } T_{k} \\ X & : & \text { state vector } \\ X_{0} & : & \text { initial state vector } \\ X_{f} & : & \text { final state vector } \\ \lambda & : & \text { costate vector } \\ \Delta t_{i, j} & : & \text { half duration of the } j^{\text {th }} \text { thrust of thruster } i\end{array}$

\section{Introduction}

For proximity operations purposes, spacecraft have to make rendezvous to a target point or to their nominal operating position. Thus, it is necessary to design an accurate control strategy in order to fulfil the rendezvous mission requirements. To this end, spacecraft are equipped with electric and/or chemical thrusters, and studies about effective numerical solutions for minimum-fuel rendezvous problems, dating back to the sixties, ${ }^{5,12)}$ are still on progress nowadays for both types of propulsion. ${ }^{4,6,14)}$

When using chemical thrusters, the thrusts are so high that the thrusting durations are short in comparison to the orbital period and the thrusts can therefore be idealised as impulsive manoeuvres (instantaneous change of velocity without change of position). As opposed to chemical thrusters, electric thrusters require a longer thrusting duration leading to the low-thrust class of rendezvous problems. Despite the drawbacks of necessitating complex power management and the very low level of the thrust, electric propulsion is nowadays a viable alternative to the chemical propulsion system thanks to the saving of on-board fuel. ${ }^{10)}$ Reducing the on-bord fuel mass leads to an increase of the payload. ${ }^{11)}$ However, as the available electrical power is first allocated to the payload, the use of electric thrusters raises some operational constraints.

For a satellite moving in an inverse square gravitational field (keplerian assumption), the relative dynamics with respect to a reference point evolving in a circular orbit can be expressed by the the Hill-Clohessy-Wiltshire equations. In this framework, the minimum-fuel rendezvous problem is naturally recast as a fuel optimal control problem with linear dynamics. Operational constraints on the propulsion system induce to add control constraints that are hard to handle with classical approaches. For instance a minimum dwell time must stand between two consecutive firings and the thrusters must have an on-off profile. Although the dynamics can be stated in a simple manner, taking the operational constraints into account requires to design a dedicated numerical approach to solve the OCP.

Between the existing numerical methods, one have to distinguish the direct and the indirect methods. Direct methods as the collocations methods ${ }^{1,9)}$ rely on a parametrisation of hte decision profiles (input and/or state) and the discretisation of the constraints. The infinite-dimensional optimisation problem (OCP) is thus recast as a finite-dimensional nonlinear programming problem. Indirect approaches for solving OCP rely on the application of the Pontryagin Maximum 
Principle (PMP). First order necessary conditions are derived in order to end up with a Two-Point Boundary Value Problem (TPBVP), that has to be solved with a Newton-Raphson algorithm or a shooting method. These approaches have complementary drawbacks (sub-optimality and a lack of precision for the former while the latter is hard to initialise and less flexible), it is not unusual to resort to hybrid methods that combine the two approaches: the solution of a direct method is used as an initialisation to the solution of the TPBVP. ${ }^{2,8)}$

However, adding the previously mentioned operational constraints on the control makes the hybrid approach not completely effective, justifying to resort to a two-step decomposition approach as mentioned in reference 7) for a geostationary station keeping problem. The difficult operational constraints are first removed from the OCP so that it can be numerically solved with an hybrid method. In a second step, an equivalent trajectory is sought in order to fulfil the operational constraints. However, in order to meet the final rendezvous constraints, numerous parameters are to be tuned, what makes the searching of the optimal switching times feasible but time consuming. ${ }^{7)}$

Assuming that no singular arcs exist, it may be shown that the minimum fuel optimal control law has an on-off profile and an ensuing difficulty is to find the optimal switching times respecting the operational constraints. In the reference 15), a method for solving switched system based on the parametrisation of the switching sequence is presented. Noting that a system with an on-off control profile exhibits a switching sequence from the system whith off control control to the system with on control, and vice-versa, it is possible to apply the switched systems technique from 15) to optimise the commutation times.

The contribution of this paper is to apply the optimisation of the commutation times for the fixed-time fuel optimal rendezvous problem using a method coming from the switched systems theory. The proposed method is embedded in a more general framework consisting in a three-step decomposition method. The first one solves an OCP without the operational constraints and gives the optimal number of thrusts per thrusters whereas the second step manages to enforce the operational constraints and thus furnishes a feasible sequence of thrusts but with still some room to improve the overall consumption by optimising the commutation times. The benefits of using the proposed optimisation of the switching times are illustrated on a numerical low-thrust rendezvous problem example. This example clearly shows the improvements due to the addition of the proposed third step when comparing to the results obtained with the two ones of the reference 7 ).

\section{Modelling in the Switched Systems Framework}

\subsection{Rendezvous problem statement}

A satellite in its terminal phase of rendezvous with a target orbiting the Earth on the geostationary Earth orbit. Assuming that the satellite is only submitted to a central attraction field, its position and velocity vectors can be computed relatively to the fictitious geostationary mission operation point in a local LVLH frame (see Fig. 1). In such a coordinate frame, the state vector consists in the vector of relative positions and velocities:

$$
X(t)=\left[\begin{array}{llllll}
x(t) & y(t) & z(t) & \dot{x}(t) & \dot{y}(t) & \dot{z}(t)
\end{array}\right]^{T}
$$

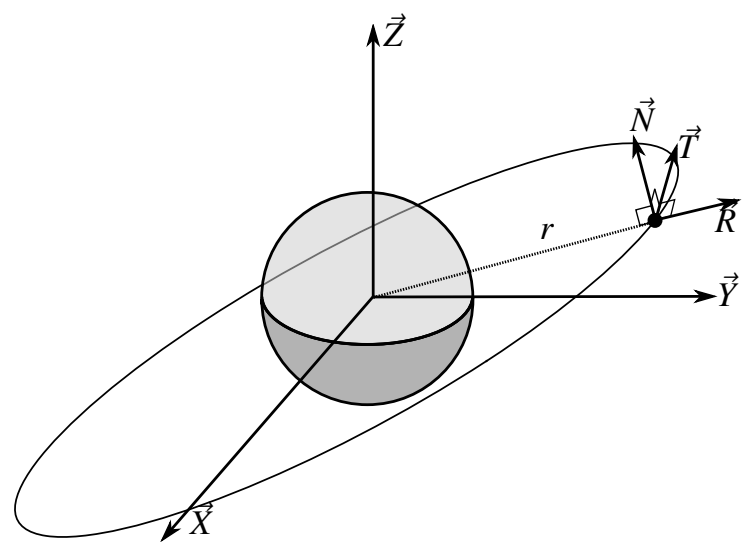

Figure1.: Local orbital frame $R T N$.

The satellite is supposed to be equipped with an electric thruster on each face allowing 6 degrees of freedom control. The control vector has thus six components and reads:

$$
u=\frac{F_{\max }}{m n^{2}}\left[\begin{array}{llllll}
u_{R} & u_{T} & u_{N} & u_{-R} & u_{-T} & u_{-N}
\end{array}\right]^{T} \in\left[0 ; \frac{F_{\max }}{m n^{2}}\right]^{6},
$$

where $m$ is the satellite mass, $n$ its mean motion and $F_{\text {max }}$ the maximum thrust level. Each component of the thrust vector is thus supposed to be either 0 or 1 .

As the reference point is located on a circular keplerian orbit, the relative motion of the satellite is given by the HillClohessy-Wiltshire equations: ${ }^{3)}$

$$
\dot{X}(t)=\underbrace{\left[\begin{array}{cc}
\mathbf{0}_{3} & \mathbf{I}_{3} \\
A_{1} & A_{2}
\end{array}\right]}_{A} X(t)+\underbrace{\left[\begin{array}{cc}
\mathbf{0}_{3} & \mathbf{0}_{3} \\
n^{2} \mathbf{I}_{3} & -n^{2} \mathbf{I}_{3}
\end{array}\right]}_{B} u(t),
$$

where $\mathbf{0}_{3}$ is the $3 \times 3$ null matrix, $\mathbf{I}_{3}$ is the $3 \times 3$ identity matrix and:

$$
A_{1}=\left[\begin{array}{ccc}
3 n^{2} & 0 & 0 \\
0 & 0 & 0 \\
0 & 0 & -n^{2}
\end{array}\right], \quad A_{2}=\left[\begin{array}{ccc}
0 & 2 n & 0 \\
-2 n & 0 & 0 \\
0 & 0 & 0
\end{array}\right]
$$

The thrusters are supposed to be on-off thrusters. Therefore the thrust profile is modelled as a rectangular signal that is parametrized by the date $t_{i, j}$ corresponding to the middle instant of the thrust and by its half width duration denoted $\Delta t_{i, j}$ as depicted on Fig. 2. Operational thruster propulsion system constraints must be taken into account while solving the rendezvous problem:

(i) thrusters cannot have simultaneous thrusts;

(ii) a thrust must last at least $T_{l}: 2 \Delta t_{i, j} \geqslant T_{l}$;

(iii) two successive thrusts of a given thruster must be separated of an interval of latency equal to $T_{s}$;

(iv) two thrusts of two different thrusters must be separated by an interval of latency equal to $T_{d}$. 


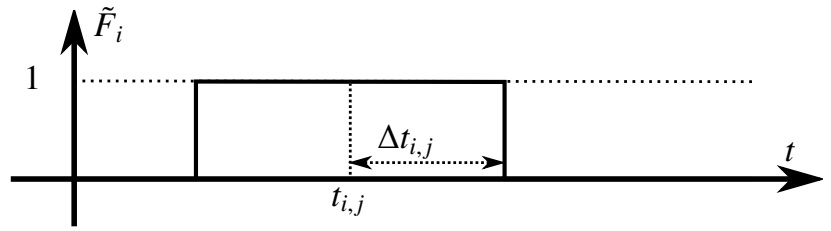

Figure2.: Parametrization of the $j^{\text {th }}$ thrust.

These constraints are technological constraints inherent to the use of an electric propulsion system. Note that these strong requirements induced by the use of electric propulsion come from the industrial partner and aerospace manufacturer Thales Alenia Space when defining the geostationary SK problem. First, the restricted available on-board power prevents two thrusters from being active simultaneously. Secondly, the minimum time latency between two thruster firings is imposed in order to allow an efficient battery recharge. ${ }^{13)}$

\subsection{Formulation of the problem in the switched systems framework}

The control profile of the system can be decomposed in $K$ intervals $T_{k}$ with constant control vector $U_{k}$. Each interval must verify:

$$
\bigcap_{i=1}^{K} T_{k}=\emptyset \text { and } \bigcup_{i=1}^{K} T_{k}=\left[t_{0}, t_{f}\right],
$$

and the control vector $U_{k}$ must be one of the $2^{6}=64$ admissible control vectors:

$$
\frac{U_{k}}{U_{\max }} \in\left\{\left[\begin{array}{c}
0 \\
\vdots \\
0 \\
0
\end{array}\right],\left[\begin{array}{c}
0 \\
\vdots \\
0 \\
1
\end{array}\right],\left[\begin{array}{c}
0 \\
\vdots \\
1 \\
0
\end{array}\right],\left[\begin{array}{c}
0 \\
\vdots \\
1 \\
1
\end{array}\right], \ldots,\left[\begin{array}{c}
1 \\
\vdots \\
1 \\
1
\end{array}\right]\right\},
$$

with $U_{\max }=\frac{F_{\max }}{m n^{2}}$.

The disjunction constraint (i) imposes to eliminate the control vectors for which more than one thruster is active. Hence, the admissible control vectors are only the 7 remaining ones:

$$
\frac{U_{k}}{U_{\max }} \in\left\{\left[\begin{array}{l}
0 \\
0 \\
0 \\
0 \\
0 \\
0
\end{array}\right],\left[\begin{array}{l}
1 \\
0 \\
0 \\
0 \\
0 \\
0
\end{array}\right],\left[\begin{array}{l}
0 \\
1 \\
0 \\
0 \\
0 \\
0
\end{array}\right],\left[\begin{array}{l}
0 \\
0 \\
1 \\
0 \\
0 \\
0
\end{array}\right],\left[\begin{array}{l}
0 \\
0 \\
0 \\
1 \\
0 \\
0
\end{array}\right],\left[\begin{array}{l}
0 \\
0 \\
0 \\
0 \\
1 \\
0
\end{array}\right],\left[\begin{array}{l}
0 \\
0 \\
0 \\
0 \\
0 \\
1
\end{array}\right]\right\} .
$$

These 7 admissible control vectors define the seven possible modes that satisfy constraint (i). It is thus possible to express the system dynamics (3) by separating the several possible modes. On the interval $T_{k}$, the system dynamics are:

$$
\dot{X}(t)=\left\{\begin{array}{l}
A X(t), \\
\text { or } \\
A X(t)+B V_{1}, \\
\text { or } \\
A X(t)+B V_{2}, \\
\text { or } \\
\vdots \\
A X(t)+B V_{6},
\end{array}\right.
$$

with $V_{i}$ being a $6 \times 1$ zero vector with a 1 at the $i^{\text {th }}$ position. By extension, it is possible to write by extension $U_{0}$ as the $6 \times 1$ zero vector.
With the considerations introduced above, all the dynamics functions:

$$
f_{i}(X)=A X+B V_{i}, \quad i \in 0, \ldots, 6
$$

can represent a subsystem of the overall system. Hence, the commutation from $U_{k}$ to $U_{k+1}$ between the intervals $T_{k}$ and $T_{k+1}$ can be viewed as a commutation between two of the seven subsystems. In a switched system framework, the system dynamics can be written as:

$$
\text { for } t \in T_{k}, \exists i_{k} \in\{0, \ldots, 6\}, \dot{X}(t)=f_{i_{k}}(X(t)) \text {, }
$$

where the following equality holds : $U_{k}=V_{i_{k}}$.

\section{Constrained Optimal Control Problem}

\subsection{Optimal control problem statement}

The rendezvous problem has to be solved on the fixedtime interval $\left[t_{0}, t_{f}\right]$ and $P_{i}$ denotes the number of thrusts for thruster $i$ in this time interval. If $\left(t_{i, k}\right)_{k=1 \ldots P_{i}}$ is the ordered sequence of firing times for thruster $i$, the constraints (iii) and (iv) may be expressed as:

$$
\left|t_{i, k}-t_{j, l}\right|-\left(\Delta t_{i, k}+\Delta t_{j, l}\right) \geqslant K_{i, j},
$$

for $k=1 \ldots P_{i}$ and $l=1 \ldots P_{j}$, where $K_{i, j}=T_{s}$ if $i=j$ (constraint (iii)) and $K_{i, j}=T_{d}$ otherwise (constraint (iv)).

Some additional constraints are used in order to prevent the firing of thrusters before $t_{0}$ or after $t_{f}$ :

$$
t_{i, j}-\Delta t_{i, j} \geqslant t_{0} \text { and } t_{i, j}+\Delta t_{i, j} \leqslant t_{f}
$$

The aim is to perform a minimum-fuel fixed-time rendezvous from the initial state $X\left(t_{0}\right)=X_{0}$ to the final state $X\left(t_{f}\right)=X_{f}$. The performance index to be minimized is:

$$
J=J(u)=\int_{t_{0}}^{t_{f}} \sum_{i=1}^{6}\left|u_{i}(t)\right| d t .
$$

The fuel-optimal rendezvous problem is thus recast as the following Optimal Control Problem (OCP):

\section{Problem 1}

$$
\begin{gathered}
\min _{u(t)} J(u)=\int_{t_{0}}^{t_{f}} \sum_{i=1}^{6}\left|u_{i}(t)\right| d t \\
\text { s.t. }\left\{\begin{array}{l}
\dot{X}(t)=A X(t)+B u(t) \\
X\left(t_{0}\right)=X_{0}, X\left(t_{f}\right)=X_{f}, \\
2 \Delta t_{i, j} \geqslant T_{l}, \forall i=1, \ldots, 6, \forall j=1, \ldots, P_{i}, \\
t_{i, j}-\Delta t_{i, j} \geqslant t_{0}, \forall i=1, \ldots, 6, \forall j=1, \ldots, P_{i}, \\
t_{i, j}+\Delta t_{i, j} \leqslant t_{f}, \forall i=1, \ldots, 6, \forall j=1, \ldots, P_{i}, \\
\left|t_{i, k}-t_{j, l}\right|-\left(\Delta t_{i, k}+\Delta t_{j, l}\right) \geqslant K_{i, j}, \\
u_{l}(t) \in[0,1],
\end{array}\right.
\end{gathered}
$$

with $u_{l}$ standing for the six components $u_{R}, \ldots, u_{-N}$ of the control vector and $P_{i}, i=1, \ldots, 6$ being the number of thrusts for each thrusters. 


\subsection{Framework of the proposed method}

The Problem 1 is an optimal control problem with constraints on the control that is difficult to solve because the operational constraints (i) - (iv) define a non connex admissible set. Moreover, the number of firings for each thruster is not known in advance, turning the Problem 1 into a non linear integer optimisation problem. A two-step decomposition method has been proposed in 7), whose steps are briefly explained here for completeness. For the first step, the operational constraints are removed so that the Problem 1 is transformed to:

\section{Problem 2}

$$
\begin{aligned}
& \min _{u(t)} J(u)=\int_{t_{0}}^{t_{f}} \sum_{i=1}^{6}\left|u_{i}(t)\right| d t \\
& \text { s.t. }\left\{\begin{array}{l}
\dot{X}(t)=A X(t)+B u(t) \\
X\left(t_{0}\right)=X_{0}, X\left(t_{f}\right)=X_{f}, \\
u_{l}(t) \in[0,1] .
\end{array}\right.
\end{aligned}
$$

The Problem 2 can be solved by an indirect method. Defining the Hamiltonian:

$$
\mathcal{H}(X, \lambda, u)=\sum_{i=1}^{6}\left|u_{i}(t)\right|+\lambda(t)^{T}(A X(t)+B u(t)),
$$

with $\lambda \in \mathbb{R}^{6}$ the costate vector, the Pontryagin Maximum Principle states that the optimal control is given by:

$$
u^{*}=\underset{u}{\operatorname{argmin}} \mathcal{H}\left(X^{*}, \lambda^{*}, u\right)
$$

Assuming that no singular arc exists, the optimal control can be restated as:

$$
u^{*}=-\operatorname{sign}\left(B^{T} \lambda^{*}\right)
$$

and is thus a bang-bang control. The operational constraint of having an on-off control profile is automatically satisfied thanks to Eq. (18). In the case where optimal singular arcs are present, strict optimality for the genuine problem would not be reached due to this constraint on the propulsion system.

Substituting $u^{*}$ into the Hamilton canonical equations leads to the following Two-Point Boundary Value Problem (TPBVP):

Problem 3 Find the functions $t \mapsto X(t)$ and $t \mapsto \lambda(t)$ solutions of :

$$
\begin{gathered}
\left\{\begin{array}{l}
\dot{X}(t)=A X(t)-B \operatorname{sign}\left(B^{T} \lambda^{*}\right), \\
\dot{\lambda}(t)=-A^{T} \lambda(t),
\end{array}\right. \\
\text { with the boundary conditions : } \\
\left\{\begin{array}{l}
X\left(t_{0}\right)=X_{0}, X\left(t_{f}\right)=X_{f}, \\
\lambda\left(t_{0}\right) \text { and } \lambda\left(t_{f}\right) \text { free, }
\end{array}\right.
\end{gathered}
$$

given by the transversality conditions.

Well-known methods to solve the TPBV problem 3 are given by multiple or simple shooting methods which, despite their precision, are in general difficult to initialise, in particular for on-off optimal control profiles. One way to deal with this difficulty is to use the solution given by a direct method as initialisation for the solution of the TPBVP $3{ }^{2}$ ) Note that the solution of the first step is a thrust profile that does not respect the operational constraints. However, as a by-product, this profile gives us candidates for the optimal numbers of thrusts per thrusters $P_{i}, i=1, \ldots, 6$.

The second step aims at enforcing the operational constraints by applying a consumption based equivalence scheme (see Problem 4) or an effect based equivalence scheme (see Problem 5). the objective of these two equivalence schemes is to preserve the structure and the consumption of the solution obtained at the first step.

\section{Problem 4}

$$
\begin{aligned}
\min _{t_{i, j}, \Delta t_{i, j}} \sum_{i=1}^{6}\left(\left\|u_{\mathrm{BVP}, i}(t)\right\|_{1}\right. & \left.-\sum_{j=1}^{P_{i}} \Delta t_{i, j}\right) \\
& +\left(X\left(t_{f}\right)-X_{f}\right)^{T} Q\left(X\left(t_{f}\right)-X_{f}\right),
\end{aligned}
$$

such that the constraints (ii),(iii) and (iv) are satisfied, and where the final position $X\left(t_{f}\right)$ implicitly depends on the parameters $t_{i, j}$ and $\Delta t_{i, j} . u_{\mathrm{BVP}}(t)$ is the control solution of Problem 3.

\section{Problem 5}

$$
\min _{t_{i, j}, \Delta t_{i, j}}\left(X(2 P+1)-X_{f}\right)^{T} Q\left(X(2 P+1)-X_{f}\right),
$$

such that the constraints (ii),(iii) and (iv) are satisfied, and where the final position $X\left(t_{f}\right)$ implicitly depends on the parameters $t_{i, j}$ and $\Delta t_{i, j}$.

The control profile of the second step provides a firing sequence respecting the operational constraints, but whose commutation times still have to be optimised. Therefore, based on the switched systems theory, ${ }^{15)}$ a third step performing the optimisation of the consumption by refining the dates $t_{k}$ is now proposed.

\subsection{Setting up the OCP in the Switched Systems Frame- work}

In order to transform the OCP 1 in the switched systems framework, it is necessary to know in advance the number of firings per thrusters and a thrusting sequence. The former will be recovered from the first step whereas the latter will be recovered from the second step.

A system with a bang-bang control profile can be considered as a switched system with two subsystems : one whose control is on and the other whose control is off. It is therefore possible to use the modelling of the rendezvous problem introduced in Section 2.2. where the system has been decomposed into seven subsystems: one for a coasting arc and one for the firing arc for each thruster. In this modelling, at most one thruster can be on at each time.

Rewritting the dynamics of the system:

$$
\text { for } t \in T_{k}, \exists i_{k} \in\{0, \ldots, 6\}, \dot{X}(t)=f_{i_{k}}(X(t)) \text {, }
$$

it can be seen that the control is now known depending on the active interval $T_{k}$. Indeed, the control vector $U_{k}$ is either 0 or $U_{\max }$ for the thruster corresponding to the active subsystem on $T_{k}$. The unknown are now the commutation times between each subsystem. 
If $P$ is the optimal number of thrusts, the number of switching times is $2 P$ and the number of intervals on which the control is constant is $2 P+1$. Denoting $t_{k}$ the switching times, it is possible to write $t_{f}=t_{2 K+1}$ as if the final time were the last commutation time.

In order to satisfy the operational constraints (iii) and (iv), each firing arc must be separated by a coasting arc. Therefore, assuming that the first arc is a coasting arc, the intervals $T_{2 k+1}$ are always coasting arcs and the intervals $T_{2 k}$ are firing arcs whose length must respect the constraints:

- $t_{2 k+1}-t_{2 k} \geqslant T_{s}$ if the coasting arc lies between two firing arcs of the same thruster,

- $t_{2 k+1}-t_{2 k} \geqslant T_{d}$ if the coasting arc lies between two firing arcs of two different thrusters.

The operational constraint (ii) is written as:

$$
t_{2 k}-t_{2 k-1} \geqslant T_{l}
$$

The cost function for the fuel minimisation is written in terms of the commutation times as:

$$
J(u)=J\left(\left\{t_{k}\right\}\right)=\sum_{k=1}^{P}\left(t_{2 k}-t_{2 k-1}\right) \frac{F_{\max }}{m n^{2}} .
$$

The application of the necessary conditions on the state and the costate vectors for Problem 1 where $J(u)=J\left(\left\{t_{k}\right\}\right)$ yields:

$$
\left\{\begin{array}{l}
\dot{X}^{*}=\left(\frac{\partial \mathcal{H}}{\partial \lambda^{*}}\right)^{T}=f_{i_{k}}\left(X^{*}(t)\right) \text { for } t \in T_{k}, \\
\dot{\lambda}^{*}=-\left(\frac{\partial \mathcal{H}}{\partial X^{*}}\right)^{T}=-\left(\frac{\partial f_{i_{k}}}{\partial X^{*}}\right) \text { for } t \in T_{k} .
\end{array}\right.
$$

Due to the transversality conditions, the costate vector must satisfy:

$$
\lambda\left(t_{0}\right) \text { and } \lambda\left(t_{f}\right) \text { free. }
$$

As the structure of the optimal control is supposed to be known in advance, the parametrisation by the switching times avoid the use of the transversality conditions on $\lambda^{*}$ for imposing the final state constraint $X\left(t_{f}\right)=X_{f}$. Therefore, it is necessary to add a penalisation of the gap between the actual final state and the target state and to modify the performance index. The new performance index to be minimised is thus:

$$
\begin{aligned}
\tilde{J}=\tilde{J}\left(\left\{t_{k}\right\},\right. & X(2 P+1))=\sum_{k=1}^{P}\left(t_{2 k}-t_{2 k-1}\right) \frac{F_{\max }}{m n^{2}} \\
& +\left(X(2 P+1)-X_{f}\right)^{T} Q\left(X(2 P+1)-X_{f}\right),
\end{aligned}
$$

where the matrix $Q$ is defined by:

$$
Q=\left[\begin{array}{cc}
\mu_{x} \mathbf{I}_{3} & \mathbf{0}_{3} \\
\mathbf{0}_{3} & \mu_{v} \mathbf{I}_{3}
\end{array}\right]
$$

\section{Problem 6}

$$
\begin{gathered}
\min _{\left\{t_{k}\right\}, X(2 P+1)} \tilde{J}\left(\left\{t_{k}\right\}, X(2 P+1)\right)=\sum_{k=1}^{P}\left(t_{2 k}-t_{2 k-1}\right) \frac{F_{\text {max }}}{m n^{2}} \\
\quad+\left(X(2 P+1)-X_{f}\right)^{T} Q\left(X(2 P+1)-X_{f}\right) \\
\text { s.t. }\left\{\begin{array}{l}
\dot{X}(t)=f_{i_{k}}(X(t)), \text { with } i_{k} \in\{0, \ldots, 6\} \text { for } t \in T_{k}, \\
X\left(t_{0}\right)=X_{0}, \\
t_{2 k}-t_{2 k-1} \geqslant T_{l}, t_{2 k+1}-t_{2 k} \geqslant \alpha,
\end{array}\right.
\end{gathered}
$$

where $\alpha=T_{s}$ in case of two successive thrusts of a given thruster or $\alpha=T_{d}$ in case of two thrusts of two different thrusters.

\section{Optimisation of the Switching Sequence in the Switched System Framework}

The Problem 6 is an OCP for a switched system. The reference 15) has developed a technique in order to solve such problems. A difference between the problem solved by 15) and the Problem 6 lies in the fact that the commutation times of the bang-bang control have been interpreted as the switching times from a subsystem whose control is 0 to a subsystem whose control is $U_{\max }$, and vice versa. Assuming that the commutation sequence, i.e. the ordered sequence of active subsystems, is known in advance, the optimal switching times are sought and can be found by applying the method described in ${ }^{15)}$ based on a switched system framework with parametrisation of the switching times. The appropriate control vector $U_{k}$ is applied on each interval $[k, k+1]$.

The idea of the technique described in 15) is to parametrise the switching times. Changing the time variable as:

$$
t=t_{k}+\Delta_{k}(\tau-k) \text { if } t \in\left[t_{k}, t_{k+1}\right]
$$

with $\Delta_{k}=t_{k+1}-t_{k}$, the switching times become parameters of the optimal control problem and the system dynamics (22) can be expanded and rewritten as:

$$
\frac{\partial X(\tau)}{\partial \tau}= \begin{cases}A X \Delta_{2 k-2} & \text { if } \tau \in[2 k-2,2 k-1], \\ \left(A X+\frac{F_{\max }}{m n^{2}} B U_{k}\right) \Delta_{2 k-1} & \text { if } \tau \in[2 k-1,2 k], \\ \vdots & \text { if } \tau \in[2 P, 2 P+1],\end{cases}
$$

for $k=1, \ldots, P$. The state vector can be now considered as a function of the new time variable $\tau$ and of the switching times $t_{k}: X=X\left(\tau,\left\{t_{k}\right\}\right)$.

The problem to be solved with the introduced change of time coordinates is thus the following one:

The OCP to be solved is thus given by: 


\section{Problem 7}

$$
\begin{gathered}
\min _{\left\{t_{k}\right\}, X(2 P+1)} \tilde{J}\left(\left\{t_{k}\right\}, X(2 P+1)\right)=\sum_{k=1}^{P}\left(t_{2 k}-t_{2 k-1}\right) \frac{F_{\text {max }}}{m n^{2}} \\
+\left(X(2 P+1)-X_{f}\right)^{T} Q\left(X(2 P+1)-X_{f}\right) \\
\text { s.t. } \\
\frac{\partial X(\tau)}{\partial \tau}= \begin{cases}A X \Delta_{2 k-2} & \text { if } \tau \in[2 k-2,2 k-1], \\
\left(A X+\frac{F_{\max }}{m n^{2}} B U_{k}\right) \Delta_{2 k-1} & \text { if } \tau \in[2 k-1,2 k], \\
\vdots & \text { if } \tau \in[2 P, 2 P+1], \\
A X \Delta_{2 P} & \\
X(0)=X_{0}, & \\
\Delta_{2 k-1} \geqslant T_{l}, \Delta_{2 k} \geqslant \alpha, & \end{cases}
\end{gathered}
$$

where $\alpha=T_{s}$ in case of two successive thrusts of a given thruster or $\alpha=T_{d}$ in case of two thrusts of two different thrusters.

As the structure of the optimal control is known, the first order necessary optimality conditions would not give any useful information, and the optimal state trajectory can be obtained by propagating the system dynamics (31).

If the overall switching times optimisation problem is solved with a descent method, it is necessary to compute the derivative of the performance index with respect to the parametrised switching times. Computing $\frac{\partial \tilde{J}}{\partial t_{k}}$ will require the computation of $\frac{\partial x}{\partial t_{k}}$, obtained by differentiation of the system dynamics (31)

The derivative of the performance index with respect to the switching times are given by:

$$
\frac{\partial \tilde{J}}{\partial t_{2 l-1}}=-\frac{F_{\max }}{m n^{2}}+2\left(Q\left(X(2 P+1)-X_{f}\right)\right)^{T} \frac{\partial X(2 P+1)}{\partial t_{2 l-1}}
$$

$$
\frac{\partial \tilde{J}}{\partial t_{2 l}}=+\frac{F_{\max }}{m n^{2}}+2\left(Q\left(X(2 P+1)-X_{f}\right)\right) \frac{\partial X(2 P+1)}{\partial t_{2 l}}
$$

and the derivatives of the state vector with respect to the switching times are:

$$
\frac{\partial}{\partial \tau}\left(\frac{\partial X}{\partial t_{2 l-1}}\right)=\left\{\begin{array}{l}
\Delta_{2 k-2} A \frac{\partial X}{\partial t_{2 l-1}} \text { if } \tau \in[2 k-2,2 k-1], \\
\Delta_{2 k-1} A \frac{\partial X}{\partial t_{2 l-1}} \text { if } \tau \in[2 k-1,2 k], \\
\Delta_{2 l-2} A \frac{\partial X}{\partial t_{2 l-1}}+A X \text { if } \tau \in[2 l-2,2 l-1], \\
\Delta_{2 l-1} A \frac{\partial X}{\partial t_{2 l-1}} \\
\quad-\left(A X+\frac{F_{\max }}{m n^{2}} B U_{l}\right) \text { if } \tau \in[2 l-1,2 l],
\end{array}\right.
$$

and

$$
\frac{\partial}{\partial \tau}\left(\frac{\partial X}{\partial t_{2 l}}\right)=\left\{\begin{array}{l}
\Delta_{2 k-2} A \frac{\partial X}{\partial t_{21}} \text { if } \tau \in[2 k-2,2 k-1], \\
\Delta_{2 k-1} A \frac{\partial X}{\partial t_{2 l}} \text { if } \tau \in[2 k-1,2 k], \\
\Delta_{2 l-2} A \frac{\partial X}{\partial t_{2 l}} \\
\quad+\left(A X+\frac{F_{\max }}{m n^{2}} B U_{l}\right) \text { if } \tau \in[2 l-1,2 l], \\
\Delta_{2 l-1} A \frac{\partial X}{\partial t_{2 l}}-A X \text { if } \tau \in[2 l, 2 l+1] .
\end{array}\right.
$$

The optimisation of the performance index and the switching times can be computed using any non linear solver. The equations (33) - (36) can be useful if a descent algorithm is used to perform the optimisation.

\section{Numerical Results}

In this section, the proposed methodology is applied on a low-thrust rendezvous involving a satellite of mass 4850 $\mathrm{kg}$ supposed to be equipped with 6 thrusters, one on each side. This satellite has to fly from its initial position $X_{0}=$ $\left[\begin{array}{llllll}5 & 10 & 10 & 0 & 0 & 0\end{array}\right]^{T}$ (the positions are given in $\mathrm{km}$ and the velocities in $\mathrm{km} /$ day) to its rendezvous target $X_{f}=$ $\left[\begin{array}{llllll}0 & 0 & 0 & 0 & 0 & 0\end{array}\right]^{T}$ in a fixed period of time $\left(t_{f}-t_{0}=\right.$ 1 day).

The process to obtain the optimal structure of the control profile satisfying the operational constraints (i) - (iv) is: ${ }^{7)}$

- remove the operational constraints,

- solve the OCP obtained with an hybrid method (see Problem 2),

- solve the consumption based equivalence (CBE) with the operational constraints and the modified cost function with a penalisation of the final state (see Problem 4),

- solve the effect based equivalence (EBE) as described in Problem 5.

Once the order of the firing thrusters is known by means of the modified CBE or the EBE, the proposed optimisation technique can optimise the switching times.

Fig. 3 shows the positions and the velocities of the satellite after solving the modified CBE and the EBE problems on one hand, and on the other hand the position and velocity of the satellite after solving Problem 7. The parameters for the final rendezvous constraint are : $\mu_{x}=100, \mu_{v}=0.001$. Fig. 4 shows the trajectories in the the $(x, y)$ plane. The norm of the final position shows that the use of the proposed technique allows to optimise the switching times computed by the modified $\mathrm{CBE}$ and EBE schemes so that the resulting trajectory comes closer to the final target with less fuel consumption (see Table 1).

Fig. 5 shows the control profile solution of the modified CBE problem, the EBE problem the control profile after having optimised the switching times using Problem 7.

Table 2 displays the computational cost for each of the three steps solved in the decomposition method and the overall computation time obtained on an Intel Inside Core i5vPro 

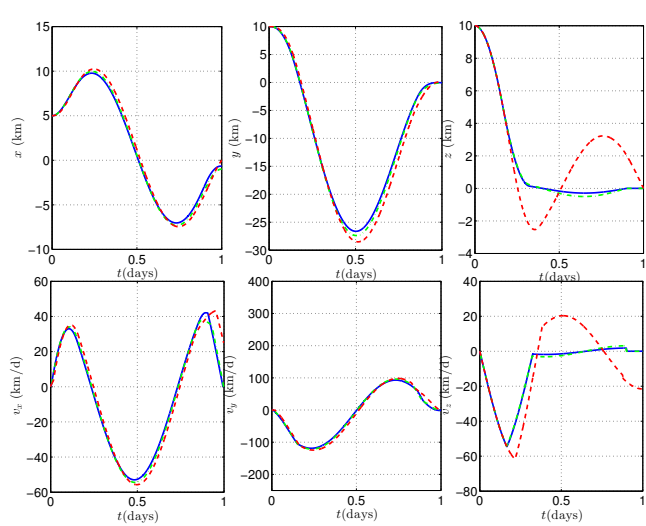

Figure 3.: Satellite positions and velocities:

-: solution of the modified CBE Problem 4,

$-\cdots$ : solution of the EBE Problem 5,

- -: solution of Problem 7.

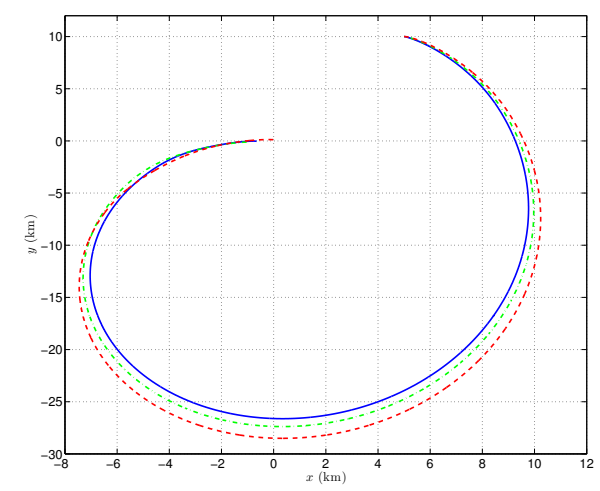

Figure4.: In plane positions:

-: solution of the modified CBE Problem 4,

---: solution of the EBE Problem 5,

- -: solution of Problem 7.

using Matlab R2014. A distinction is made for the step 2 between the consumption based equivalence scheme or the effect based equivalence scheme.

Table1.: Final position $\left\|X\left(t_{f}\right)\right\|$, final velocity $\left\|V\left(t_{f}\right)\right\|$ and consumption for the modified CBE Problem 4, the EBE Problem 5 and the proposed technique (Problem 7).

\begin{tabular}{cccc}
\hline Problem & Problem 4 & Problem 5 & Problem 7 \\
\hline$\left\|X\left(t_{f}\right)\right\|(\mathrm{m})$ & 658.0 & 1008 & 114.9 \\
$\left\|V\left(t_{f}\right)\right\|(\mathrm{m} / \mathrm{s})$ & $5.10^{-4}$ & $4.10^{-4}$ & 0.39 \\
consumption $(\mathrm{m} / \mathrm{s})$ & 2.051 & 2.052 & 1.881 \\
\hline
\end{tabular}

\section{Conclusion}

In this paper, the switched system framework is used in order to optimise the commutation sequence for a fixed-time minimum-fuel rendezvous problem whose control presents an on-off profile. In this case, the system can be naturally separated into two subsystems, depending on the value of the control: on or off. After the parametrisation of the switching times, a descent algorithm can be used to minimise the

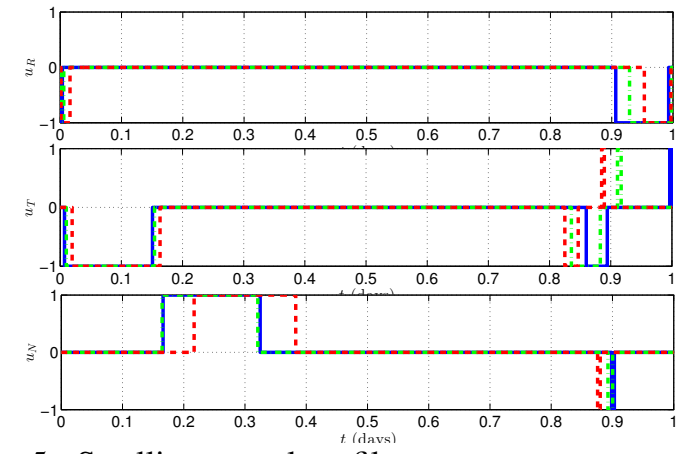

Figure5.: Satellite control profiles:

-: solution of the modified CBE Problem 4,

---: solution of the EBE Problem 5,

- -: solution of Problem 7 ,

$u_{M}=u_{+M}-u_{-M}$ with $M \in\{R, T, N\}$.

Table2.: Computational cost for each of the three steps and for the overall decomposition method.

\begin{tabular}{ccc}
\hline Step & Problems & Computational time (s) \\
\hline 1 & 3 & 73.38 \\
\hline \multirow{2}{*}{2} & 4 & 76.20 \\
& 5 & 200.88 \\
\hline 3 & 7 & 879.71 \\
\hline \multirow{2}{*}{ Total } & $3+4+7$ & 1029.29 \\
& $3+5+7$ & 1153.97 \\
\hline
\end{tabular}

overall fuel consumption as well as the distance to the target. As the proposed method requires to know beforehand the optimal thrusters firing sequence, a two-step decomposition method is used in advance. The optimisation of the switching times allow to overcome the drawbacks of the previous steps. Hence it can be considered as the third step of the decomposition. An improvement of the presented results would be to extend the technique to time varying and nonlinear systems.

\section{References}

1) Betts, J. T.: Survey of Numerical Methods for Trajectory Optimization, Journal of Guidance, Control, and Dynamics, 21 (1998), pp. 193-207.

2) Bulirsch, R., Nerz, E., Pesch, H. J., and von Stryk, O.: Combining Direct and Indirect Methods in Optimal Control: Range Maximization of a Hang Glider, in Optimal Control, ISNM International Series of Numerical Mathematics 111. Birkhäauser Basel, 1993, pp. 273-288 .

3) Clohessy, W. H. and Wiltshire, R. S.: Terminal Guidance System for Satellite Rendezvous, Journal of the Aerospace Sciences, 27 (1960), pp. 653-658.

4) Dankanich, J. W.: Low-Thrust Mission Design and Application, 46th AIAA/ASME/SAE/ASEE Joint Propulsion Conference \& Exhibit (2010).

5) Euler, E. A.: Optimal Low-Thrust Rendezvous Control, AIAA Journal 7 (1969), pp. 1140-1144.

6) Feng, W., Wang, B., Yang, K., and Zhao, D.: Cooperative Rendezvous between two Spacecraft under Finite Thrust, CEAS Space J., 9 (2017), pp. 227-241.

7) Gazzino, C., Arzelier, D., Losa, D., Louembet, C., Pittet, C., and Cerri, L.: Optimal Control for Minimum-Fuel Geostationary Station Keeping of Satellites Equipped with Electric Propulsion, IFAC-PapersOnLine 49 (2016), pp. 379-384. 
8) Grimm, W., and Markl, A.: Adjoint Estimation from a Direct Multiple Shooting Method, Journal of optimization theory and applications 92 (1997), pp. 263-283.

9) Hull, D. G.: Conversion of Optimal Control Problems into Parameter Optimization Problems, Journal of Guidance, Control, and Dynamics 20 (1997), pp. 57-60.

10) Jahn, R. G. and Choueiri, E. Y.: Electric Propulsion, Encyclopedia of Physical Science and Technology, 5 (2002), pp. 125-141.

11) Koppel, C. R.: Advantages of a Continuous Thrust Strategy from a Geosynchronous Transfer Orbit, using High Specific Impulse Thrusters, 14th International Symposium on Space Flight Dynamics - ISSFD XIV, Feb., 1999, pp. 8-12.

12) Lawden, D. F.: Optimal Trajectories for Space Navigation, Butterwort edition, London, 1963

13) Losa, D., Lovera, M., Marmorat, J.-P., Dargent, T., and Amalric, J.: Station Keeping of Geostationary Satellites with On-Off Electric Thrusters, Computer Aided Control System Design, 2006 IEEE International Conference on Control Applications, 2006 IEEE International Symposium on Intelligent Control, 2006 IEEE, pp. 2890-2895, 2006.

14) Serra, R., Arzelier, D., Rondepierre, A., and Calvet, J.-L.: Analytical Optimal Solutions of Impulsive Out-of-plane Rendezvous around Elliptic Orbits, IFAC-PapersOnLine 19 (2014), pp. 2231-2236.

15) Xu, X., and Antsaklis, P. J.: Optimal Control of Switched Systems Based on Parameterization of the Switching Instants, IEEE Trans. Automatic Control, 49 (2004), pp. 2-16. 